\title{
Experimental Measurements and Numerical Simulations of Particle Transport and Distribution in Ventilated Rooms
}

\author{
Z. ZHANG ${ }^{\mathrm{a}}$, Q. CHEN ${ }^{\mathrm{a}}$ \\ ${ }^{a}$ School of Mechanical Engineering, Purdue University, 585 Purdue Mall, West \\ Lafayette, IN 47907-2088, USA
}

\begin{abstract}
Prediction of particle dispersion and distribution in a room is very important for creating and maintaining a healthy indoor environment. The present study used a CFD program with a Lagrangian particle tracking method to predict particle dispersion and concentration distribution in ventilated rooms. Since the Lagrangian method could generate great uncertainty in particle concentration calculations, this study first investigated such uncertainty using a statistical approach. It was found that the stability of the concentration solution became well controlled as a sufficient number of particles were analyzed. Although the overall computational cost was considerable, the numerical results agreed well with associated experimental data. In all cases studied, particle size distribution was monodisperse, and particle diameter ranged from 0.31 to $4.5 \mu \mathrm{m}$. Particle deposition rate was neglected, and particles were hence removed only by the ventilation system. Thus the particle removal performance of different ventilation systems can be evaluated. Three ventilation systems have been studied, including ceiling and side wall supply systems and an underfloor air distribution (UFAD) system. It was found that the UFAD system had a better particle removal performance than the ceiling and side wall supply systems in the study. However, resuspended particles at the floor level can still cause problems in an underfloor air distribution system.
\end{abstract}

Keywords: Fine particles; Indoor air; Underfloor air distribution; Ventilation systems; Lagrangian particle tracking, CFD

\section{Introduction}

Suspended particulate matter can serve as nuclei and carriers for airborne viruses and bacteria, resulting in the spread of diseases. In addition, fine particles themselves can deposit in the lungs and cause respiratory diseases. As people spend about $90 \%$ of their lifetime indoors (Klepeis et al., 2001; Aliaga and Winqvist, 2003), indoor particulate matter can have great impact on human health. Thus, a good understanding of particle transport is crucial for creating healthy indoor environments.

Many particle studies have been conducted in the last decade. Nazaroff (2004) summarized the state-of-the-art modeling techniques used to study indoor particle dynamics, such as infiltration, filtration, indoor source emission, and deposition. His review was based on the assumption of a well mixed condition of room air and particles.

\footnotetext{
* Corresponding author. Tel.: +765 496 7562; E-mail address: yanchen@purdue.edu
} 
This assumption could fail because perfect mixing is difficult. In order to accurately design a healthy indoor environment, it is important to consider spatial distributions of particles.

Computational fluid dynamics (CFD) is a powerful tool for studying particle dispersion and spatial distribution with either the Eulerian or Lagrangian method. The Eulerian method treats particles as a continuum and solves the conservation equations for particle phase. On the other hand, the Lagrangian method emphasizes the individual behavior of each particle and determines particle trajectories based on the equation of motion. Each method has its own pros and cons. Summaries of merits and recent developments for each method were discussed throughout the literature, e.g., Shirolkar et al. (1996) and Loth (2000).

In indoor particle studies, each method earns its own popularity depending on the research objective. The Eulerian method is widely used to predict particle concentration distributions in rooms (Murakami et al., 1992; Shimada et al., 1996; Holmberg and Chen, 2003; Zhao et al., 2004a). Generally, those simulations agree well with experimental data, although remarkable discrepancies exist in some studies. If the particle motion and dispersion history is of interest, the Lagrangian method is more attractive (Lu et al., 1996; Zhang and Chen 2004; Zhao et al., 2004b). By tracking and analyzing a large number of trajectories, these studies successfully predicted the particle dynamics and the temporal development of particle dispersion. Some recent studies even claimed that the Lagrangian approach could be more accurate than the Eulerian method in predicting pollutant dispersion and distribution (Loomans and Lemaire, 2002; Riddle et al., 2004). In fact, little work has been done to validate and explore the capability of Lagrangian particle tracking in predicting detailed particle concentration distribution in rooms. This study aims to validate the Lagrangian particle model as a useful tool in calculating particle concentration in ventilated rooms.

Furthermore, the present study applied the validated model to analyze ventilation system performance for particle removal in indoor air. Previous studies showed that underfloor air distribution (referred as UFAD hereafter) system is better at removing indoor gas contaminants as compared to other typical ventilation systems (e.g., Chen et al., 2004). As particulate matter often behaves quite different from gas phase pollutants, it is interesting to study whether or not the UFAD is also the most efficient for particle removal. Thus this investigation conducted a detailed analysis of the particle dispersion in a room with UFAD and compared the results to rooms with ceiling supply and side wall supply systems.

\section{CFD modeling}

\subsection{The turbulent airflow model}

This study used the Eulerian method to simulate the airflow field. The interaction between fluid phase and discrete phase was assumed to be one way, and the impact from discrete phase to fluid phase was negligible. This is feasible since the particle volume 
fraction is sufficiently small. Thus the airflow field is independent on the particulate phase and could be determined in advance.

The Reynolds averaged Navier-Stokes (RANS) equations along with the standard k- $\varepsilon$ turbulence model were employed to predict the incompressible turbulent airflow in a room. The general form of the governing equations can be generalized as:

$$
\frac{\partial(\rho \Phi)}{\partial \mathrm{t}}+\operatorname{div}\left(\rho \overrightarrow{\mathrm{u}} \Phi-\Gamma_{\Phi, \text { eff }} \operatorname{grad}(\Phi)\right)=\mathrm{S}_{\Phi}
$$

where $\Phi$ represents the independent variables: time averaged velocity components $\bar{u}_{i}$ $(\mathrm{i}=1,2,3)$, turbulent kinetic energy $\mathrm{k}$, dissipation rate of turbulent kinetic energy $\varepsilon$, and enthalpy $H$. When $\Phi$ is unity, the equation represents the conservation of mass. Note that $\overrightarrow{\mathrm{u}}$ is the Reynolds averaged air velocity vector, and $\mathrm{t}$ is time. Expressions for the effective diffusivity $\Gamma_{\Phi, \text { eff }}$ and the source term $S_{\Phi}$ for each variable and the corresponding empirical numbers are described by Launder and Spalding (1972).

The CFD program solves Eq. (1) in the form of discretized algebraic equations. The discretization scheme used for all variables except the pressure term was the second order upwind scheme. For pressure a staggered scheme called PRESTO! was selected (FLUENT, 2005). Finally, this study used the SIMPLE algorithm to couple pressure and velocity.

\subsection{Discrete phase particle model}

In addition to the influence from the flow pattern, particle dynamic behaviors are highly dependent on particle size and size-related forces. Particle sizes are generally categorized into three modes (Nazaroff, 2004): ultrafine (smaller than $0.1 \mu \mathrm{m}$ ); accumulation $(0.1$ $2 \mu \mathrm{m}$ ); and coarse (larger than $2 \mu \mathrm{m}$ ). This study mainly focused on particles in the accumulation mode. Particles in this mode have a low deposition rate and make up a large portion of indoor particle concentration.

\subsubsection{Equations for particle motion and dynamics}

The Lagrangian particle tracking method is used to calculate individual trajectories by solving the momentum equation. By equating the particle inertia with external forces, the momentum equation can be expressed as:

$$
\frac{d \vec{u}_{p}}{d t}=F_{D}\left(\vec{u}-\vec{u}_{p}\right)+\frac{\vec{g}\left(\rho_{p}-\rho\right)}{\rho_{p}}+\vec{F}_{a}
$$

In Eq. (2), the left hand side represents the inertial force per unit mass $\left(\mathrm{m} / \mathrm{s}^{2}\right)$, where $\overrightarrow{\mathrm{u}}_{\mathrm{p}}$ is the particle velocity vector. The first term on the right hand side is the drag term, where $F_{D}$ is the inverse of relaxation time $\left(\mathrm{s}^{-1}\right)$; the second term represents the gravity and the 
buoyancy, where $\rho$ and $\rho_{p}$ are the density of air and the particles, respectively; and $\overrightarrow{\mathrm{F}}_{\mathrm{a}}$ stands for additional forces (per unit mass) that may be important.

In the current study, the drag force is the most significant force and it follows the Stokes drag law:

$$
\overrightarrow{\mathrm{F}}_{\mathrm{drag}}=\mathrm{F}_{\mathrm{D}}\left(\overrightarrow{\mathrm{u}}-\overrightarrow{\mathrm{u}}_{\mathrm{p}}\right)=\frac{18 \mu}{\rho_{\mathrm{p}} \mathrm{d}_{\mathrm{p}}^{2} \mathrm{C}_{\mathrm{c}}}\left(\overrightarrow{\mathrm{u}}-\overrightarrow{\mathrm{u}}_{\mathrm{p}}\right)
$$

where $\mu$ is fluid viscosity and $d_{p}$ is particle diameter. The Cunningham correction factor, denoted as $\mathrm{C}_{\mathrm{c}}$, is calculated using the following:

$$
\mathrm{C}_{\mathrm{c}}=1+\frac{2 \lambda}{\mathrm{d}_{\mathrm{p}}}\left(1.257+0.4 \mathrm{e}^{-\left(1.1 \mathrm{~d}_{\mathrm{p}} / 2 \lambda\right)}\right)
$$

where $\lambda$ is the molecular mean free path.

For the current study, other forces such as the Basset history, the pressure gradient and the virtual mass are infinitesimal and negligible compared to the drag force. The Brownian, thermophoretic and the shear induced lift (Saffman's lift) forces are generally at least two magnitudes smaller than the Stokes drag force in room air. However, some of these forces may occasionally become comparable in magnitude to the Stokesian drag force within the turbulent boundary layer ( $\mathrm{Li}$ and Ahmadi, 1992). Also, some forces may have a great impact on the deposition process (Rizk and Elghobashi, 1985; McLaughlin, 1989; Nazaroff and Cass, 1989). These forces were therefore treated as submicron particles in this study. The final form of the trajectory equation became:

$$
\frac{d \vec{u}_{p}}{d t}=F_{D}\left(\vec{u}-\vec{u}_{p}\right)+\frac{\vec{g}\left(\rho_{p}-\rho\right)}{\rho_{p}}+\vec{F}_{b}+\vec{F}_{\text {therm }}+\vec{F}_{s}
$$

where $\overrightarrow{\mathrm{F}}_{\mathrm{b}}, \overrightarrow{\mathrm{F}}_{\text {therm }}$, and $\overrightarrow{\mathrm{F}}_{\mathrm{s}}$ represent the Brownian term, the thermophoretic term and the lift term, respectively. Detailed descriptions on those forces are available in the FLUENT documentation (FLUENT, 2005).

The time averaged flow field determined the mean path of particles, while the instantaneous flow field governed each particle's turbulent dispersion from the mean trajectory. This investigation used the discrete random walk (DRW) model to simulate the stochastic velocity fluctuations in the airflow. The DRW model assumes that the fluctuating velocities follow a Gaussian probability distribution. The fluctuating velocity components $\mathrm{u}_{\mathrm{i}}^{\prime}$ have the following form:

$$
\mathrm{u}_{\mathrm{i}}^{\prime}=\zeta_{\mathrm{i}} \sqrt{\overline{\mathrm{u}_{\mathrm{i}}^{\prime 2}}}=\zeta_{\mathrm{i}} \sqrt{2 \mathrm{k} / 3}
$$


where $\mathrm{k}$ is the turbulent kinetic energy and $\zeta_{\mathrm{i}}$ is the normally distributed random number. The term $\mathrm{u}_{\mathrm{i}}^{\prime}$ remains constant during each time step. The time step was chosen so that a particle remains in the same local eddy within each particular time slot. By replacing the mean velocity $\overrightarrow{\mathrm{u}}$ with $\overrightarrow{\mathrm{u}}=\overline{\mathrm{u}}_{\mathrm{i}}+\mathrm{u}_{\mathrm{i}}^{\prime}$ in Eq. (5), each trajectory could then interact with the modeled flow field at each instance in time.

\subsubsection{Boundary conditions}

When particles reach air supply inlets or exhaust outlets, they will escape and the trajectories terminate. When reaching a rigid object, particles may either attach to or rebound from the object's surface. Particles in a ventilated room are most likely to attach to the surface since they usually cannot accumulate enough rebound energy to overcome adhesion (Hinds, 1982). It is therefore natural to terminate, or "trap", a particle trajectory after hitting a rigid surface. This treatment was adopted and used in many CFD studies of the indoor environment. As a result, for instance, Zhao et al. (2004b) reported that 60 out of every 160 trajectories for particles with a $1 \mu \mathrm{m}$ diameter deposit in a room with mixing ventilation; Béghein et al. (2005) predicted that about $13 \%$ of trajectories for particles with a $5 \mu \mathrm{m}$ diameter deposit in another ventilated cavity. However, such a deposition rate turns out to be much higher than expected in ventilated rooms (Lai and Nazaroff, 2000). This is due to the over prediction of particle-wall collision frequency, which is associated with the near wall treatment. In those simulations, the CFD grid was not sufficiently fine near the wall. Consequently, turbulence models over-predicted the turbulent kinetic energy within the viscous sublayer where turbulent energy damps out much faster (So et al., 1991). Particles in the viscous sublayer could then acquire an abnormally high fluctuating velocity normal to the wall. This could eventually lead to the over prediction of particle-wall collision frequency.

Nevertheless, the trap treatment worked well when the near wall grid was sufficiently fine, like that which was used in the DNS simulation (McLaughlin, 1989; Narayanan et al., 2003). The trap treatment, however, is not suitable for the current situation which uses a high Reynolds number k- $\varepsilon$ model. Instead of using trap treatment, this study set the restitution coefficient to a very small value. By doing so, particles were immediately stopped without being trapped after reaching a surface. When particles acquired sufficient normal velocity, they escaped from the boundary layer and became resuspended. This implies that deposition is neglected. Such manipulation may only be suitable when particle deposition rate is very low. As will be shown, this treatment worked well for small particles suspended in the room air and located far away from the wall.

\subsection{Particle concentration calculation}

The present study used FLUENT to calculate the airflow and the Lagrangian trajectories. However, the Lagrangian method does not directly calculate the particle concentration. So this study used a research program and FLUENT user-defined functions (UDF) to calculate concentration distribution from the trajectories. The research program applied 
the particle source in-cell (PSI-C) scheme to correlate the concentration with the trajectories on a computational cell basis:

$$
\overline{\mathrm{C}_{\mathrm{j}}}=\frac{\dot{\mathrm{M}} \sum_{\mathrm{i}=1}^{\mathrm{n}} \mathrm{dt}_{(\mathrm{i}, \mathrm{j})}}{\mathrm{V}_{\mathrm{j}}}
$$

where $\dot{M}$ is the number flow rate of each trajectory, $\mathrm{V}$ is the volume of a computational cell for use in concentration calculations, $\mathrm{dt}$ is the particle residence time, and subscript (i, $\mathrm{j})$ represents the $\mathrm{i}^{\mathrm{th}}$ trajectory and the $\mathrm{j}^{\text {th }}$ cell, respectively. Due to the stochastic nature of the particle tracking, the stability of a concentration calculation depends on the number of trajectories and relates to the resolution of the control volumes. In this paper, the concentration calculation was based on a predefined uniform mesh and not on the CFD mesh. The grid number of concentration mesh was on the order of several thousand, which was much less than that of the CFD mesh.

\section{Experiments for model validation}

In order to use the CFD model to analyze particle dispersion in a room, it is necessary to validate the model. Experimental data has been sought for validating the CFD model. Murakami et al. (1992) measured the airflow field and particle concentration distributions in two ventilation systems under isothermal conditions. The chamber geometries are shown in Fig. 1 with specifications listed in Table 1. Since this research provided all necessary experimental details, their work was used for model validation. However, these studies only reported concentration profiles in an interpolated contour form making a point to point comparison difficult. In addition, their isothermal experiments may not be representative and informative for displacement ventilation and UFAD systems, in which the vertical temperature stratification is an important characteristic. In such cases, experiments under non-isothermal conditions are necessary.

Therefore, this research conducted additional experiments to account for the impact of non-isothermal conditions. Experimental measurements were carried out in a full scale environmental chamber with UFAD. The chamber geometry is shown schematically in Fig. 2a. The chamber dimensions in the $\mathrm{x}, \mathrm{y}$, and $\mathrm{z}$-directions are $4.91 \mathrm{~m}, 2.44 \mathrm{~m}$, and $4.31 \mathrm{~m}$, respectively. Two air supply openings were located on the floor, and the exhaust outlet was located on the ceiling. Air was supplied to the room at a total airflow rate of $0.0944 \mathrm{~m}^{3} / \mathrm{s}$. Four heated human simulators were placed on the floor and six lamps were installed on the ceiling. Fig. $2 \mathrm{~b}$ indicates measurement locations for both airflow and particle concentration.

Before each measurement, the HVAC system was run for 6-8 hours to reach a thermally steady-state condition in the room. Air temperature and velocity were then recorded and the particle experiment proceeded. A condensation monodisperse aerosol generator (TSI 3475, TSI Inc.) was used to generate fine DEHS (Di-Ethyl-Hexyl-Sebacat) particles. DEHS is a non-soluble liquid with a density of $912 \mathrm{~kg} / \mathrm{m}^{3}$ and a low evaporation rate. 
Since most particles stay in the room for a short time period, the size change due to evaporation is negligible. Under normal operation, the generator could generate monodisperse spherical particles with a GSD less than 1.2. In our experiment, the mean particle size was about $0.7 \mu \mathrm{m}$. As shown in Fig. $2 \mathrm{~b}$, the particle source was located 0.3 $\mathrm{m}$ above the floor at position S1 or S2 (referred to as Case S1 or Case S2). Particles were introduced into the room by a carrier gas (nitrogen) at a total flow rate of $7 \times 10^{-5} \mathrm{~m}^{3} / \mathrm{s}$ ( 250 1/h). A particle counter (PC-2H QCM impactor, California Measurements Inc.) was used to measure particle concentration at the exhaust outlet. The sampling probe was positioned parallel to the mean flow direction in the duct. According to Hinds (1982), the probe size will ensure isokinetic sampling. Once this concentration became stable, another identical particle counter began taking measurements inside the room. In the experiment, the nominal concentration (steady-state concentration at the exhaust) was on the order of $10^{9}$ particles $/ \mathrm{m}^{3}$. Since this was two magnitudes higher than the background concentration, the background noise in the measurement data was considered to be negligible.

To avoid disturbing the experiment, some boundary conditions were measured separately. By reproducing the thermal-fluid conditions, the measurement of detailed boundary conditions was possible. The temperature of each surface of the human simulators was measured, although only an average surface temperature is presented here due to limited space. Air injection direction was visualized using a smoke tester. The velocity fluctuations of the supply air was monitored at 9 points above each supply inlet.

\section{Model evaluation}

Both the k- $\varepsilon$ turbulence model for fluid phase and the DRW model for particulate phase have been verified by reproducing benchmark cases (Chen, 1995; Shirolkar, 1996). The present study was used to validate the integral performance of the fluid and particle model in indoor applications. Since it is very difficult to measure the particle concentration near the wall, this study mainly compared measurements to simulation results far from surfaces. Experimental data from Murakami et al. (1992) was studied first. Fig. 3 and Fig. 4 show a comparison between the measured and simulated concentration fields. The numerical simulation reasonably depicted the characteristics of measured concentration pattern. These preliminary results illustrated that the numerical model is feasible for modeling indoor particle concentration distribution. Thus, the model was further evaluated by a more detailed comparison with our experimental data from the UFAD system setup.

For the UFAD cases, the CFD simulation used the measured boundary conditions listed in Table 2. Also, the study assumed that the supply air was discharged uniformly into the room. The incident angles were $90^{\circ}$ and $75^{\circ}$ from $-\mathrm{X}$ direction and the estimated turbulent intensity was $10 \%$ and $15 \%$ for air injection from the north and south inlets, respectively. The present CFD simulations were based on a structured grid containing a total of 389,338 control volumes. Fig. 5 shows the simulated and measured velocity and temperature profiles. The simulation agreed well with measured data. Particularly, the thermal-fluid profiles near both air supply openings are well predicted, indicating that the 
approximations for the air supply injections are reasonable. By providing well defined boundary conditions, the $\mathrm{k}-\varepsilon$ model is capable of capturing the mean flow characteristics. The ability to accurately simulate flow formed the basis of quality particle concentration prediction.

The stability of the Lagrangian method is also important since stochastic particle tracking may introduce great uncertainty into the concentration calculation. Such uncertainty must be well controlled by statistical analysis to ensure sufficient stability. The present study started the analysis with a sample size (i.e., number of trajectories) of 1000 and repeated for ten runs. The concentration was calculated based on a uniform mesh containing about 7,500 cells. The mean particle concentration $\bar{X}_{n}$ and standard deviation $s_{n}$ at each cell were then calculated. As the particle tracking obeyed the normal distribution, the student's t-distribution governed the statistics. For a $95 \%$ confidence interval, it can be stated that the true mean of the particle concentration lies in the following interval:

$$
\overline{\mathrm{C}}=\overline{\mathrm{X}}_{\mathrm{n}} \pm \mathrm{t}_{\mathrm{n}-1,0.975} \frac{\mathrm{s}_{\mathrm{n}}}{\sqrt{\mathrm{n}}}
$$

where $\mathrm{n}$ is the number of times the runs were repeated and $\mathrm{t}_{\mathrm{n}-1,0.975}$ is calculated from the t-distribution (Kreyszig, 1988).

By gradually increasing the sample size, the solution became more stable, and the length of confident interval was reduced. In the current study, a sample size was considered sufficient if at a majority of the positions the confident interval was within $\pm 10 \%$ of the mean concentration. Fig. 6 illustrates this process for Case 2. As the number of trajectories increased, the fluctuation between solutions became smaller. All curves tended to converge to the results from a study using 50,000 trajectories. A sample size of 10,000 was found to be sufficient, as its statistical data met the afore mentioned $\pm 10 \%$ criterion. The same procedure was also applied to Case 1. It was found that results became sufficiently stable at a significantly greater sample size of 50,000. Although the airflow field between the two cases was identical, Case 1 required a larger sample size than Case 2 in order to meet the same stability criterion. One possible reason is that the airflow near source S1 is more dispersive than that near source S2. If the room air is far from perfect mixing, particle source location may also have an impact on the stability of the Lagrangian method and hence the required sample size. Nevertheless, both cases required that the sample size be larger than the number of computational cells in order to be sufficient. It is therefore suggested that the statistical analysis for the Lagrangian method should start from a sample size that is at least comparable to the number of concentration cells. A smaller sample size is less likely to meet the statistical criterion.

Fig. 7 further compares numerical results with the experimental data. In most positions, the measured data is close to the numerical data within the uncertainty range. The greatest difference occurs in Case S2 at the lower parts of P5 and P6. In the experiment, the concentration fluctuation near the source was relatively strong owing to flow fluctuations from the particle generator. The difference may therefore come from the lack 
of precision either in the measurement near the source or the model used for the simulation. Due to the lack of sufficient evidence, a definite explanation of the discrepancy is difficult to establish. Despite this, the general concentration pattern is well simulated in most places.

The particle model was also used to compute the temporal development of particle concentration at the exhaust. During the Lagrangian tracking process, the particle removal rate (the number of removed particles per unit time) was recorded at the end of each time step. The ratio of the particle removal rate and the total ventilation rate approximated the average particle concentration at exhaust within each time step. As only the bulk fate of trajectories was considered in such calculation, a sample size of 1,000 trajectories could produce very stable results. Again, the numerical prediction agreed well with the experimental data as shown in Fig. 8.

The results discussed so far confirm that the Lagrangian particle tracking model performed well for the cases studied. Nevertheless, each case considered had only one particle source, and the particle generation rate was held constant. In a real indoor environment, indoor particle emission may be brief and intermittent (Long et al., 2000) and multiple sources may coexist. The present concentration model can handle such situations by simply expressing $\dot{M}$ in Eq. (7) as a function of time and space. Thus the proposed method is able to handle more complicated applications in real indoor environments.

Generally, the present numerical model performed well in the study of particle dispersion and distribution in ventilated rooms. Therefore, it is suitable to use the numerical results to compare and analyze particle dispersion behavior in rooms with various ventilation systems.

\section{Particle dispersion characteristics in different ventilation systems}

The validated particle model has been used to predict particle transport and distribution in three different ventilation systems including the UFAD and the ceiling and side wall supply systems. There is enough data to analyze the particle removal performance of each system and to make a comparison between each system.

For a UFAD system, floor level particle sources may be problematic as they can be brought up by the general upward airflow. In both cases studied, the particle source was at floor level, yet the resulting concentration distributions are still quite different as shown in Fig. 7. For Case S1, the concentration profiles are similar for all poles: higher concentration at bottom portion of the room and lower concentration at upper portion of the room. On the contrary, for Case S2, the concentration profile varies with location. The concentration is relatively uniform for the poles located far away from the source (P1-P3), while the concentration generally increases with height at P4. At P5 and P6, the concentration profile becomes S-shaped. It is also observed that the concentrations at P1 through P3 are much lower than that at P4 through P6 in Case S2, while such lateral concentration stratification is not that obvious in Case S1. 
The differences between Cases S1 and S2 reveal that particle concentration distribution is sensitive to the source location in an UFAD system. This can be attributed to the airflow pattern in the present air conditioning system. Fig. 9 shows that the airflow in the lower portion of the room is well circulated and well mixed, while it is relatively unidirectional in the upper portion of the room. The air in the lower part of the room travels to upper part mainly by upward air supply injection and human generated heat plumes. Since the flow near source S1 was directed downward, most particles were brought down to the floor level and remained in the lower zone for a relatively long time. In contrast, S2 was located near an occupant with a thermal plume so most particles were carried directly to the upper zone by human generated heat plumes. This facilitated the particle removal process. In summary, the particle removal rate is much higher for Case 2 than for Case 1 during the first several minutes after the particles were released. This is demonstrated in Fig. 8.

Source S1 represents floor level particles resuspended by occupant movement and air circulation above the floor, while source S2 simulates particles generated by occupants. It is clear that the present UFAD system is capable of efficiently removing occupant generated particles, although it may not be as efficient in removing particles resuspended at the floor level. Resuspension events, however, are short and their impact does not last long (Long et al., 2000). When such resuspension events, e.g. rigorous walking, do not occur frequently, the UFAD system performs well in the control of particle concentration. This agrees with the field assessment from offices in commercial buildings with an under floor air-conditioning system (Sekhar and Ching, 2002).

Fig. 10 illustrates the particle concentration distribution in the present UFAD system. Compared with that of the ceiling and side wall air supply systems shown in Fig. 3 and 4, each type of ventilation has its own particle removal characteristics. For the ceiling supply and UFAD systems, particle concentration is stratified both horizontally and vertically, while the particle concentration in a room with the side wall supply is rather uniform. On the other hand, the normalized average concentration for the side wall supply and UFAD systems is lower than that for the ceiling supply system. As shown in Table 3, the ceiling supply system has the highest normalized average concentration while the UFAD system has the lowest. In general, the normalized average reflects the particle removal efficiency for a ventilation system. Also, appropriate concentration stratification reduces the possibility of cross contamination. Thus when both particle concentration level and particle removal efficiency need to be considered for a ventilated room, the UFAD system is the most suitable choice among the three air supply systems.

\section{Summary and conclusions}

This investigation studied the particle transport and distribution in ventilated rooms by both experimental investigation and numerical simulation. The numerical simulation was achieved by applying the k- $\varepsilon$ turbulent model, the Lagrangian particle tracking method, and the particle source in cell scheme. The Lagrangian method was introduced, evaluated 
and then used to analyze particle dispersion in rooms with three different ventilation systems.

The Lagrangian method introduced uncertainty in the particle concentration calculation. The uncertainty level is associated with Lagrangian sample size, number of computational cells for concentration, and even particle source position in the turbulent flow. This study extracted statistical data from simulated results that used different sample sizes and particle source positions to determine the particle concentration distribution with sufficient confidence. In the UFAD cases, the initial sample size ranged from 1,000 to 50,000 trajectories, and for each sample size the calculation was repeated at least 5 times. Sample sizes of 10,000 and 50,000 were reported to be sufficient for the two UFAD cases, respectively. Although the sufficient number of trajectories can be very case dependent, it is often a good practice to start the analysis with a sample size close to the grid number of the concentration mesh.

It was shown that the numerical results reasonably agreed with experimental data for all cases studied. As a result, the numerical results could be used to investigate particle distributions in three different ventilation systems including ceiling and side wall air supply systems and the UFAD system. Airflow pattern has a significant impact on the particle concentration distributions in a room. It was found that the UFAD system has best particle removal efficiency and has the greatest potential to reduce cross contamination. Nevertheless, particles resuspended or generated at floor level cannot be easily removed by the UFAD system.

In conclusion, the Lagrangian method was found to be an effective method for the study of indoor particle transport and concentration distribution provided the solution stability was guaranteed. Therefore, statistical analysis must be exercised when employing the Lagrangian method. In general, a formal statistical analysis requires considerable computational effort, i.e., tracking a huge number of particles and repeating the process multiple times. The time consuming analysis and high computational cost might limit the application of the Lagrangian method to the study of the indoor environment. Further research is necessary to compare the Lagrangian method with other modeling methods so that its pros and cons can be better appreciated for future applications.

\section{Acknowledgement}

This research is supported by the Air Transportation Center of Excellence for Airliner Cabin Environment Research through Cooperative Agreement Number 04-C-ACE-PU002 with the Federal Aviation Administration (FAA).

\section{References}

Aliage, C. and Winqvist, K., 2003, "Commnet les femmes et les homes utilisent leurs temps-Résultats de 13 pays européens,” Eurostat, KS-NK-03-012-FR-N.

Béghein, C., Jiang, Y., and Chen, Q.Y., "Using large eddy simulation to study particle motions in a room," Indoor Air, in press. 
Chen, Q., Lau, J., and Kobayashi, N., 2004, "Floor-supply displacement ventilation system," Final Report for National Institute of Occupational Safety and Health Grant, School of Mechanical Engineering, Purdue University, West Lafayette, IN.

Chen, Q., 1995, "Comparison of different k- $\varepsilon$ models for indoor airflow computations," Numerical Heat Transfer, Part B: Fundamentals, Vol. 28, pp 353-369.

FLUENT, 2005, Fluent 6.2 User's guide, Fluent Inc., Lebanon, NH.

Hinds, W.C., 1982, Aerosol Technology: properties, behavior, and measurement of airborne particles, Wiley, New York.

Holmberg, S. and Chen, Q., 2003, "Air flow and particle control with different ventilation systems in a classroom," Indoor Air, Vol. 13, pp. 200-204.

Klepeis, N.E., Nelson, W.C., Ott, W.R., Robinson, J.P., Tsang, A.M., Switzer, P., Behar, J.V., Hern, S.C., and Engelmann, W.H., 2001, "The national human activity pattern survey (NHAPS): A resource for assessing exposure to environmental pollutants," Journal of Exposure Analysis and Environmental Epidemiology, Vol. 11 (3), pp 231-252.

Kreyszig, E., 1988, Advanced Engineering Mathematics, Wiley, New York.

Lai, A.C.K. and Nazaroff, W.W., 2000, "Modeling indoor particle deposition from turbulent flow onto smooth surfaces," Journal of Aerosol Science, Vol. 31, No. 4, pp. 463-476.

Launder, B.E. and Spalding, D. B., 1972, Lectures in Mathematical Models of Turbulence, Academic Press, London, England.

Li, A. and Ahmadi, G., 1992, "Dispersion and deposition of spherical particles from point sources in a turbulent channel flow," Aerosol Science and Technology, Vol. 16, pp. 209226.

Long, C.M., Suh, H.H., and Koutrakis, P., 2000, "Characterization of indoor particle sources using continuous mass and size monitors," Journal of the Air and Waste Management Association, Vol. 50, pp. 1236-1250.

Loomans, M., and Lemaire, T., 2002, "Particle concentration caluculations using cfd-a comparison," Proceedings of Indoor Air 2002, pp. 153-156, Edinburgh, Scotland.

Loth, E., 2000, "Numerical approaches for motion of dispersed particles, droplets, and bubbles," Progress in Energy and Combustion Science, Vol. 26, pp. 161-223. 
Lu, W., Howarth, A.T., Adam, N., and Rifat, S.B., 1996, "Modelling and measurement of airflow and aerosol particle distribution in a ventilated two-zone chamber," Building and Environment, Vol. 31 (5), pp. 417-423.

McLaughlin, J.B., 1989, “Aerosol particle deposition in numerically simulated channel flow," Physics of Fluids A, Vol. 1 (7), pp. 1211-1224.

Murakami, S., Kato, S., Nagano, S., and Tanaka, S., 1992, "Diffusion characteristics of airborne particles with gravitational settling in a convection-dominant indoor flow field," ASHRAE Transactions, Vol. 98, part 1, pp. 82-97.

Narayanan, C., Lakehal, D., Botto, L., and Soldati, A., 2003, "Mechanisms of particle deposition in a fully-developed turbulent open channel flow," Physics of Fluids, Vol. 15(3), pp. 763-775.

Nazaroff, W.W., 2004, "Indoor particle dynamics," Indoor Air, Vol. 14 (Suppl 7), pp 175-183.

Nazaroff, W.W. and Cass, G.R., 1989, "Mathematical modeling of indoor aerosol dynamics," Environmental Science and Technology, Vol. 23, No. 2, pp. 157-166.

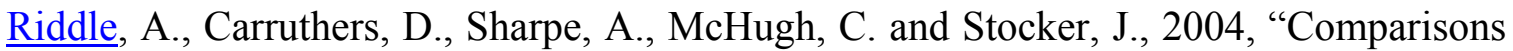
between FLUENT and ADMS for atmospheric dispersion modeling," Atmospheric Environment, Vol. 38, pp. 1029-1038.

Rizk, M.A. and Elghobashi, S.E., 1985, "The motion of a spherical particle suspended in a turbulent flow near a plane wall," Physics of Fluids, Vol. 28 (3), pp. 806-817.

Sekhar, S.C. and Ching, C.S., 2002, "Indoor air quality and thermal comfort studies of an under-floor air-conditioning system in the tropics," Energy and Buildings, Vol. 34, pp. 431-444.

Shimada, M., Okuyama, K., Okazaki, S., Asai, T., Matsujura, M., and Ishizu, Y., 1996, "Numercial simulation and experiment on the transport of fine particles in a ventilated room,” Aerosological Science and Technology, Vol. 25, pp. 242-255.

Shirolkar, J.S., Coimbra, C.F.M., and McQuay, M.Q., 1996, "Fundamental aspects of modeling turbulent particle dispersion in dilute flows," Progress in Energy and Combustion Science, Vol. 22, pp. 363-399.

So, R.M.C., Zhang, H.S., and Speziale, C.G., 1991, "Near-wall modelling of the dissipation-rate equation," AIAA Journal, Vol. 29, pp. 2069-2076.

Zhang, Z. and Chen, Q., 2004, "Numerical analysis of particle behaviors in indoor air using Lagrangian method," Roomvent Proceedings 2004, Coimbra, Portugal. 
Zhang, Z. and Chen, Q., 2005, "Particle dispersion in a room with under-floor air distribution,” Proceedings of Indoor Air 2005, Vol. III, pp. 2373-2378, Beijing, China.

Zhao, B., Zhang, Z., Li, X., and Huang, D., 2004a, "Comparison of diffusion characteristics of aerosol particles in different ventilated rooms by numerical method," ASHRAE Transactions, Vol. 110, pp. 88-95.

Zhao, B., Zhang, Y., Li, X., Yang, X., and Huang, D., 2004b, "Comparison of indoor aerosol particle concentration and deposition in different ventilated rooms by numerical method," Building and Environment, Vol. 39, Part 1, pp. 1-8. 
Table 1. Specifications of experimental chambers and conditions used by Murakami et al. (1992).

\begin{tabular}{|c|c|c|c|c|c|c|}
\hline \multirow{2}{*}{ Ventilation type } & \multicolumn{3}{|c|}{ Dimensions (m) } & \multirow{2}{*}{$\begin{array}{l}\text { Volume of air } \\
\text { change }(\mathrm{m} 3 / \mathrm{s})\end{array}$} & \multirow{2}{*}{$\mathrm{ACH}\left(\mathrm{h}^{-1}\right)$} & \multirow{2}{*}{$\begin{array}{c}\text { Particle size } \\
(\mu \mathrm{m})\end{array}$} \\
\hline & Length & Width & Height & & & \\
\hline & 5.85 & 3.29 & 2.8 & 0.64 & 43 & 0.31 \\
\hline ide wall supply & 1.05 & 0.77 & 0.49 & 0.00245 & 22 & $0.31,1,4.5$ \\
\hline
\end{tabular}

Table 2. Measured boundary conditions in the room with UFAD system.

\begin{tabular}{|c|c|c|c|c|}
\hline Surface & $\begin{array}{c}\text { Surface } \\
\text { temperature }\left({ }^{\circ} \mathrm{C}\right)\end{array}$ & Boundary & $\begin{array}{c}\text { Surface } \\
\text { temperature }\left({ }^{\circ} \mathrm{C}\right)\end{array}$ & $\begin{array}{c}\text { Heat power } \\
(\mathrm{W})\end{array}$ \\
\hline North wall $(+\mathrm{X})$ & 24.9 & Floor $(-\mathrm{Y})$ & 24 & - \\
\hline South wall $(-\mathrm{X})$ & 25.0 & Lamps $($ each) & - & 64 \\
\hline East wall $(+\mathrm{Z})$ & 25.5 & Persons (each) & 31.6 & 100 \\
\hline West wall $(-\mathrm{Z})$ & 25.3 & Supply (north) & 20.4 & - \\
\hline Ceiling $(+\mathrm{Y})$ & 25.7 & Supply (south) & 19.9 & - \\
\hline
\end{tabular}

Table 3. Normalized averaged particle concentration levels in four cases studied.

\begin{tabular}{|c|c|c|c|c|}
\hline Flow Type & \multicolumn{2}{|c|}{ UFAD } & Ceiling Supply & Mixing \\
\hline Source Location & Near inlet & Near person & Near floor in middle & Near ceiling \\
\hline ACH $(1 / \mathrm{h})$ & 7 & 7 & 43 & 22 \\
\hline$\overline{\mathrm{C}}$ & 1.07 & 0.65 & 1.72 & 0.96 \\
\hline
\end{tabular}



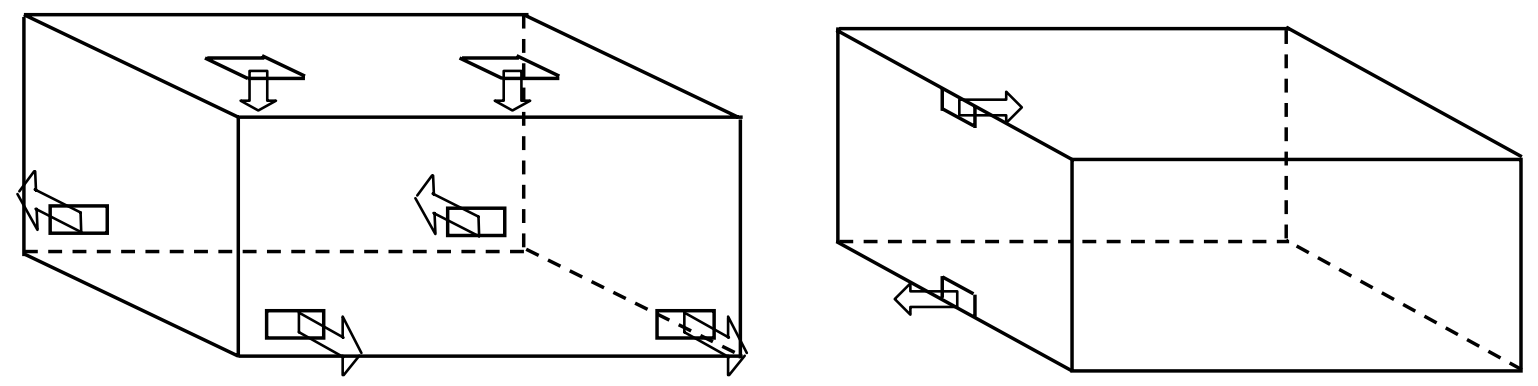

Fig. 1. A schematic view of two chambers used for full-scale experiments and simulations by Murakami et al. (1992).
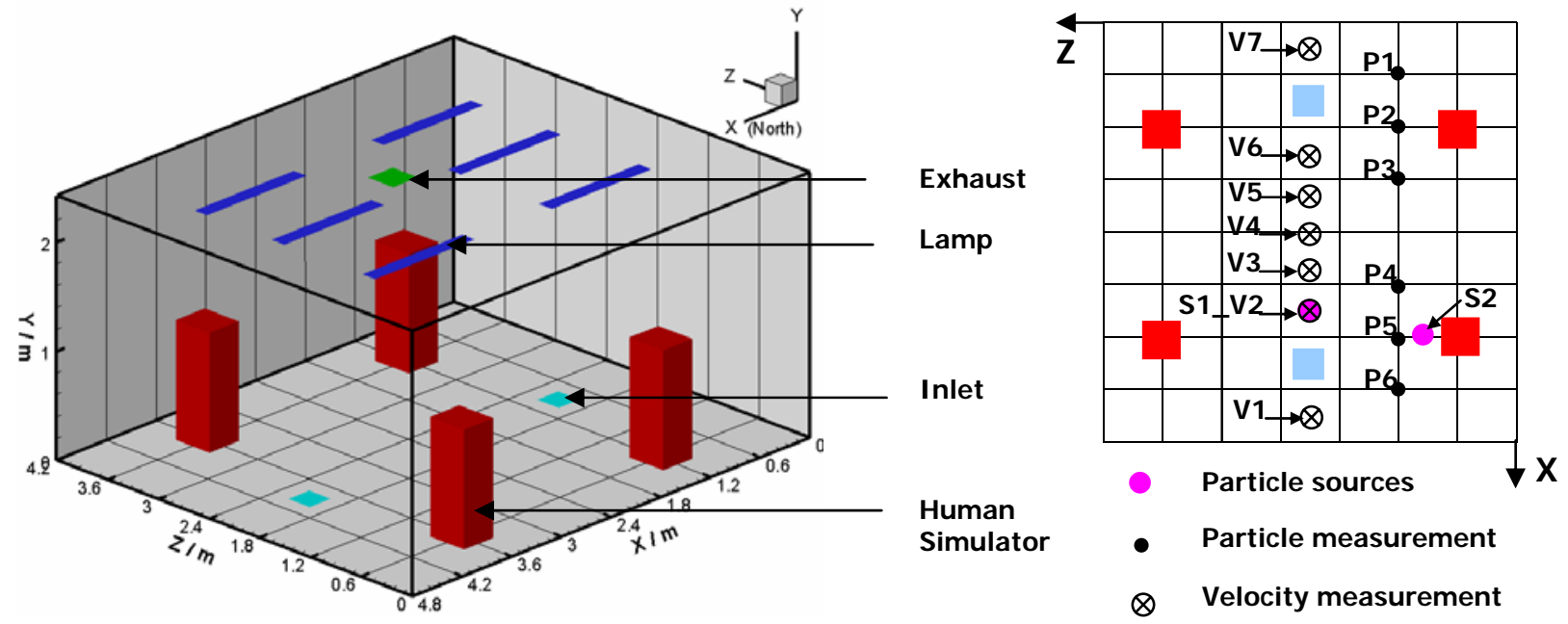

Fig. 2. A sketch of the environmental chamber configuration and experimental arrangements.

(a) 

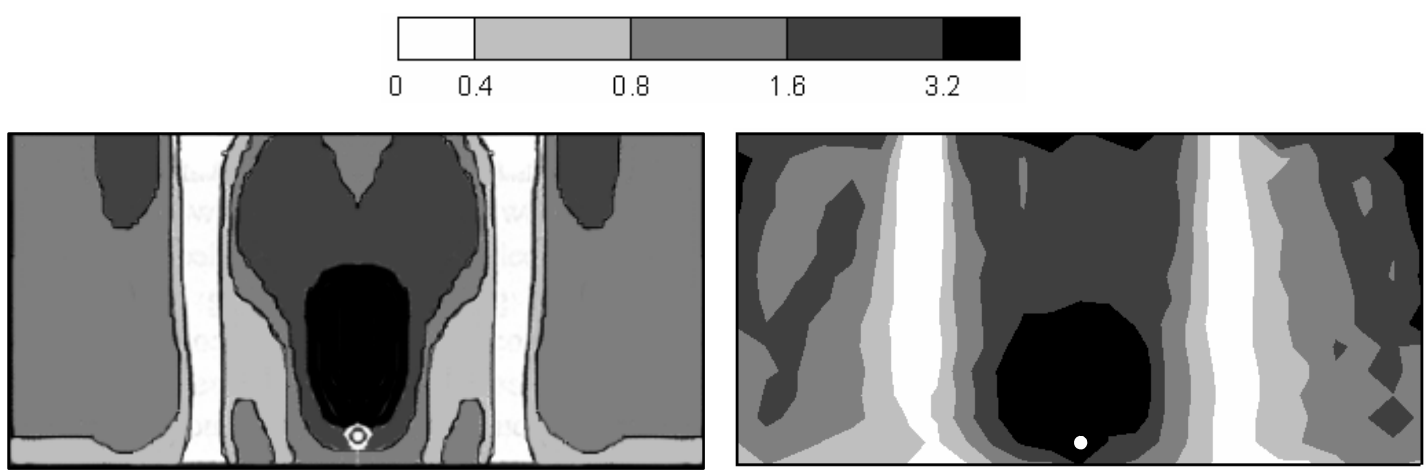

(b)
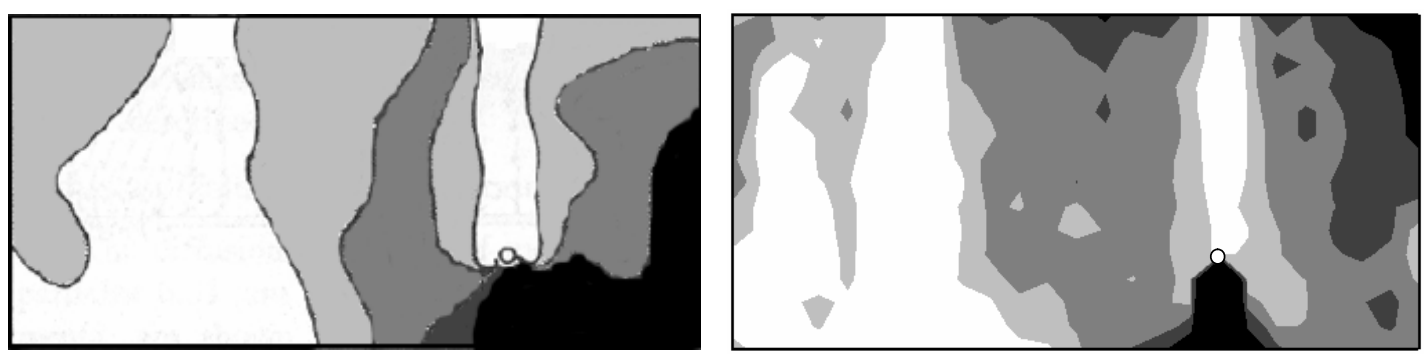

Fig. 3. Measured and predicted particle concentration distribution in the center plane of the model room with ceiling air supply: (a) source near the floor center; (b) source under one air supply (left: experimental measurements from Murakami et al. (1992); right: simulation). Particle size was $0.31 \mu \mathrm{m}$.
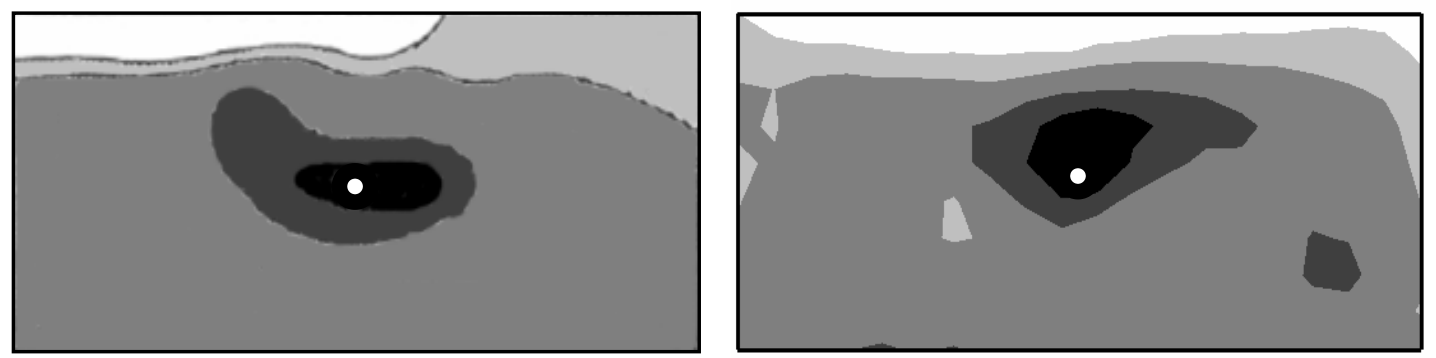

Fig. 4. Measured and predicted particle concentration distribution in the center plane of the scaled model room with side wall air supply (left: experimental measurements from Murakami et al. (1992); right: simulation). Particle size was $4.5 \mu \mathrm{m}$. For this particular case, only drag force was considered. 

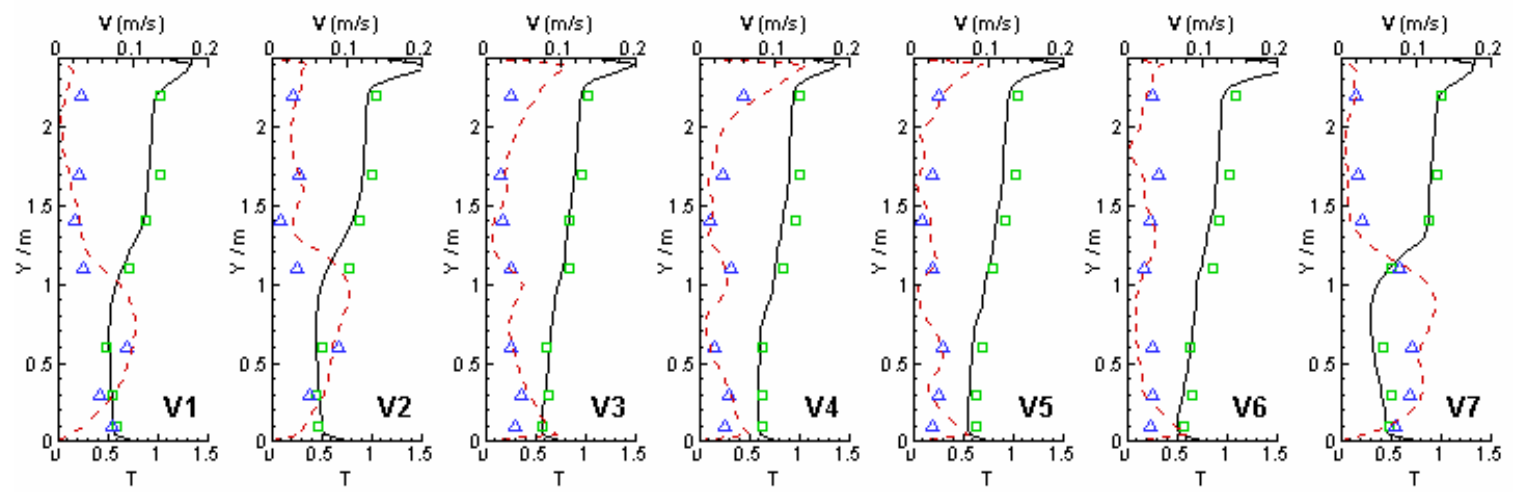

Fig. 5. Comparison of simulated and measured temperature and velocity profiles (rectangular symbols: measured temperature after normalization; triangle symbols: measured velocity; solid lines: simulated temperature; dash line: simulated velocity). Temperature is normalized as $\mathrm{T}=\left(\mathrm{T}-\mathrm{T}_{\text {supply }}\right) /\left(\mathrm{T}_{\text {exhaust }}-\mathrm{T}_{\text {supply }}\right)$.

(a)
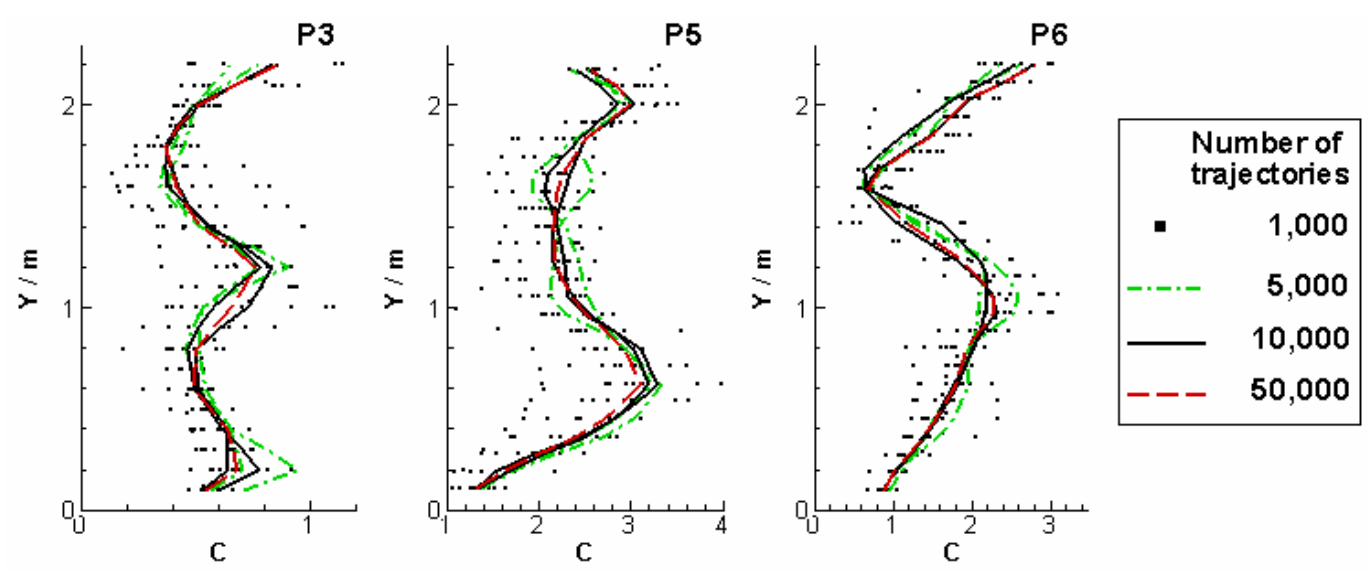

(b)
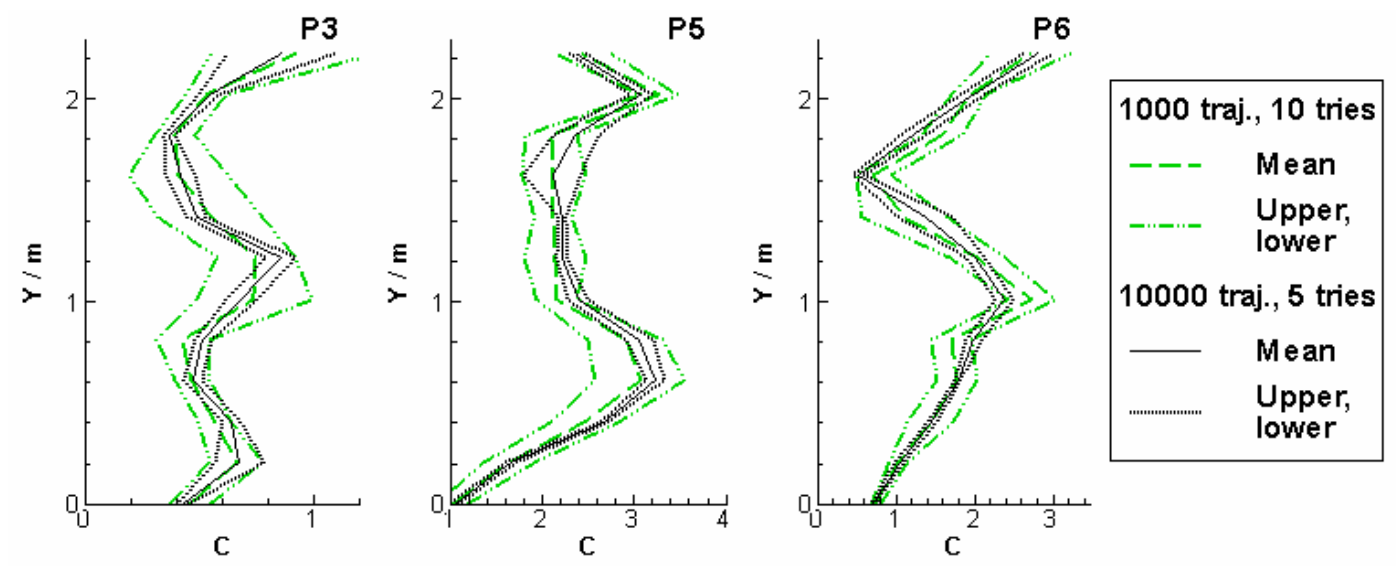

Fig. 6. An investigation of solution stability at three poles in Case 2: (a) particle concentration solutions from different sample sizes; (b) $95 \%$ confident intervals for two sample sizes, 1000 and 10000 trajectories, with respectively 10 and 5 repeats.

(a) 

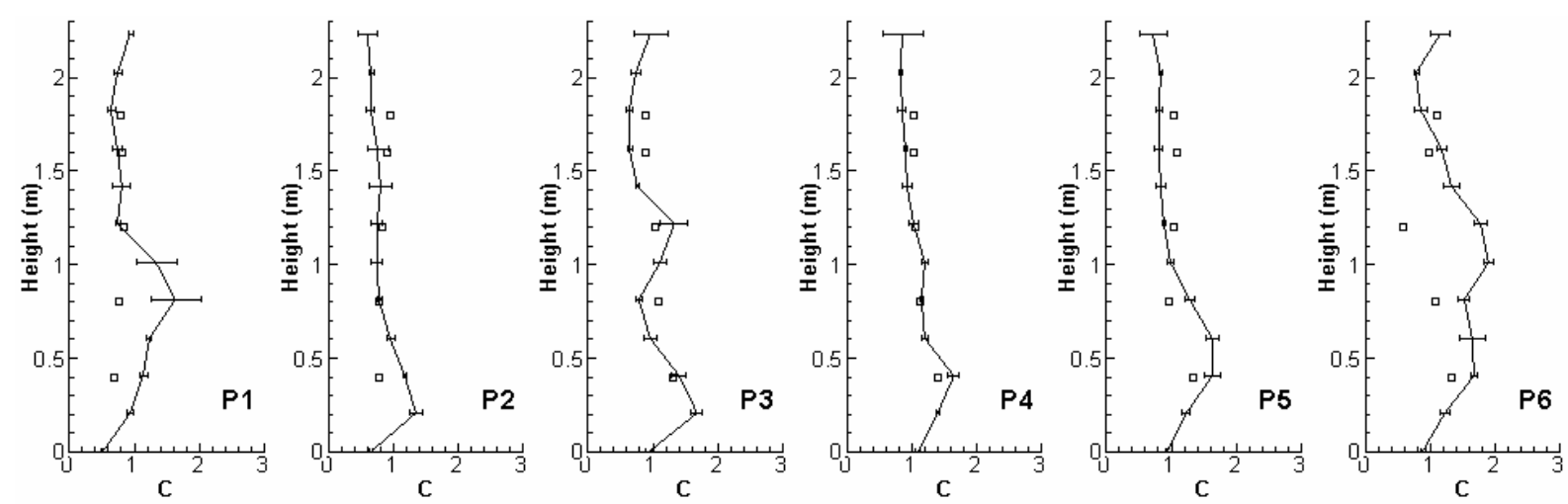

(b)
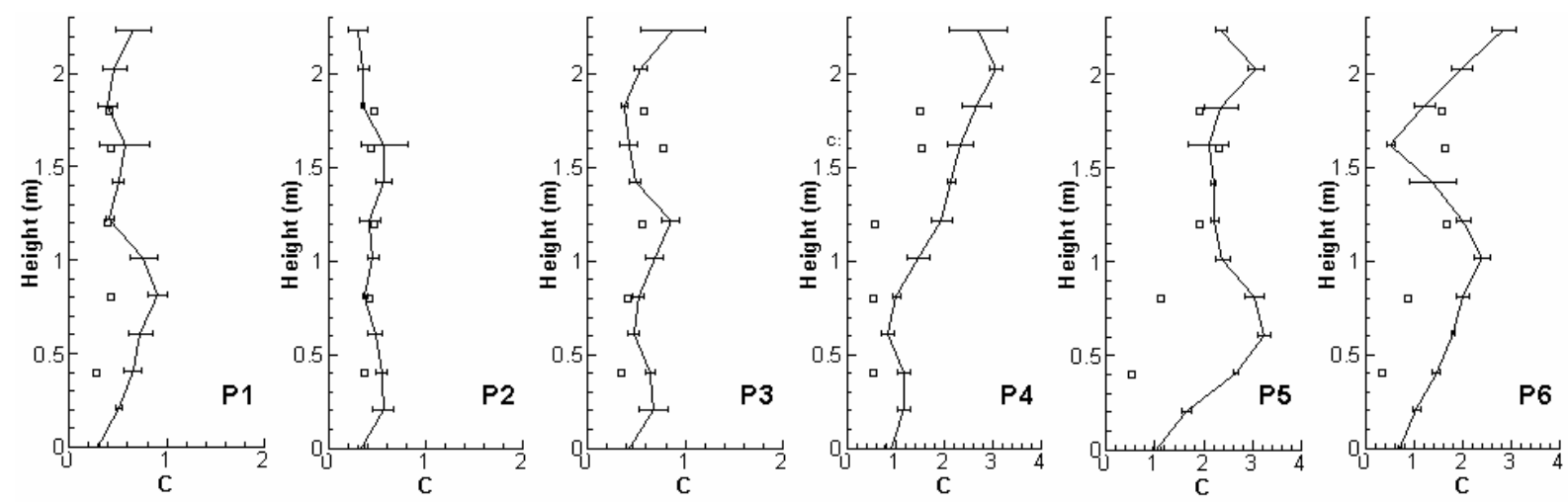

Fig. 7. A comparison of measured and simulated particle concentration: (a) Case S1 and (b) Case S2 (rectangular symbols: experimental data; solid line: simulations; error bar on solid line: $95 \%$ confident interval). The particle concentration was normalized by the steady-state nominal particle concentration at the exhaust. For Case 1 and 2, the sample sizes were 50,000 and 10,000 respectively, and number of repeats was 5 .

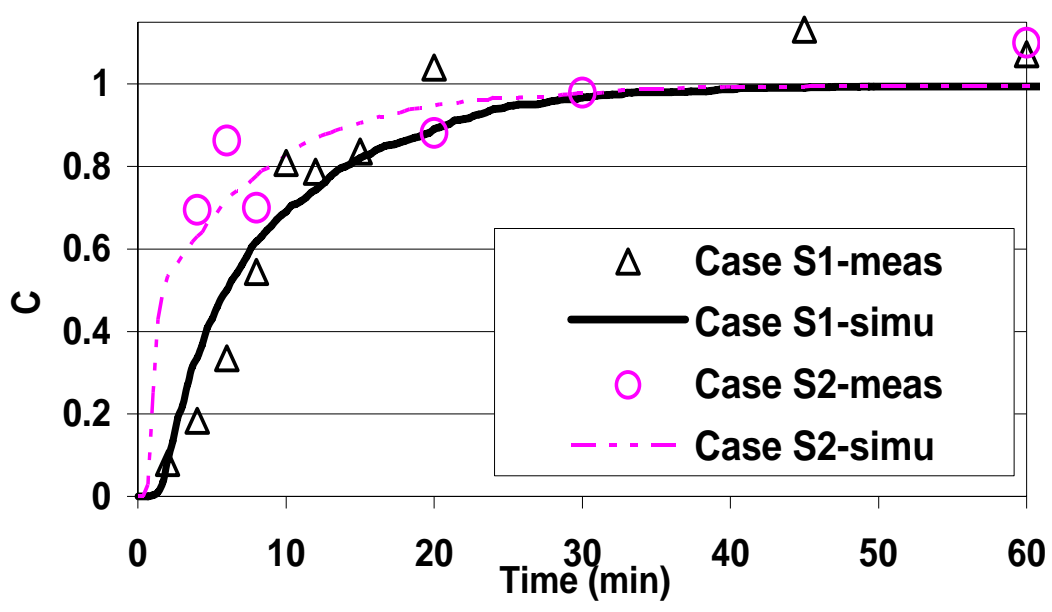

Fig. 8. Measured and simulated temporal development of normalized particle concentration at the exhaust in two UFAD cases (symbols: experimental data; curve line: simulations). 


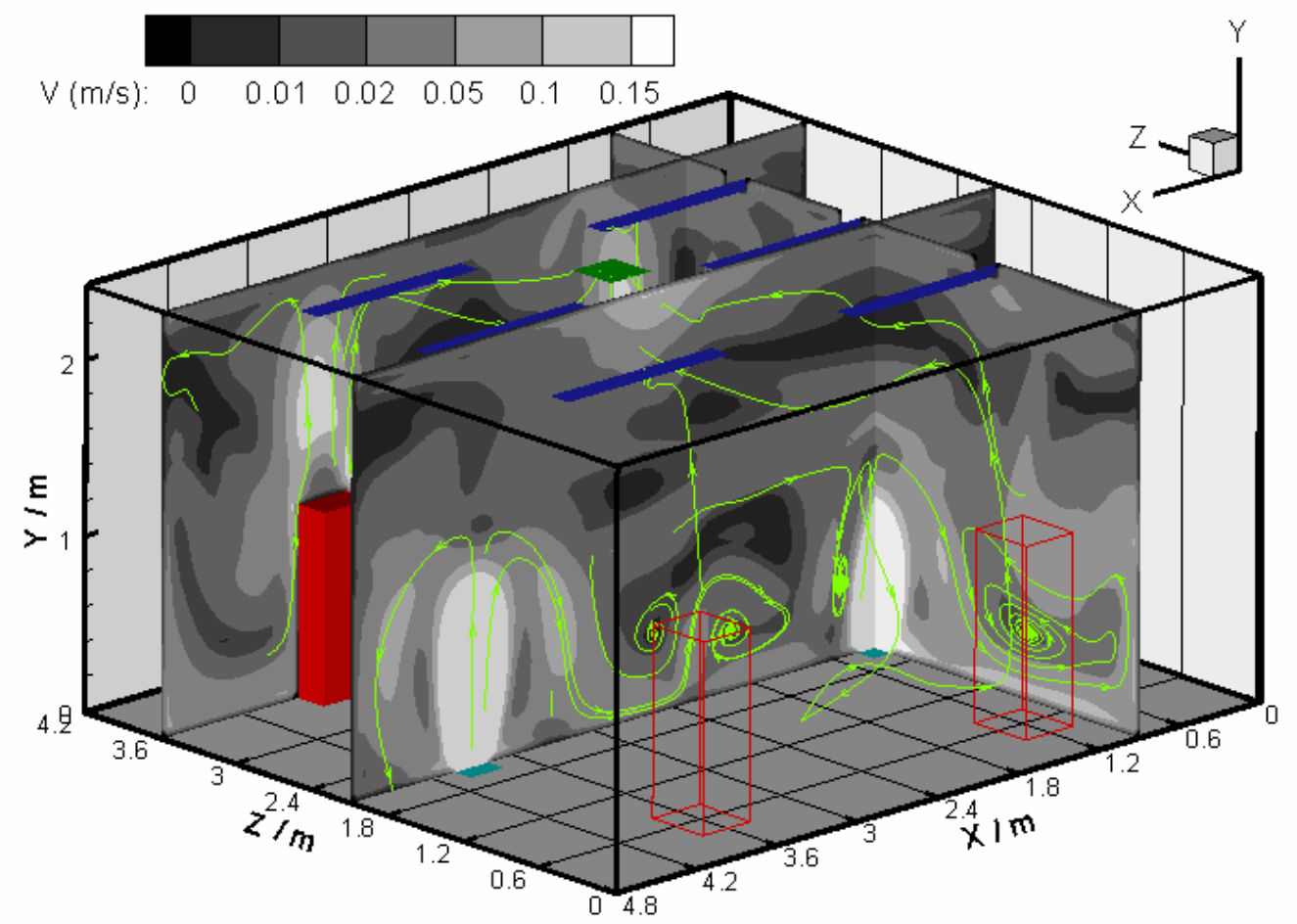

Fig. 9. Simulated airflow field in the chamber with UFAD system.

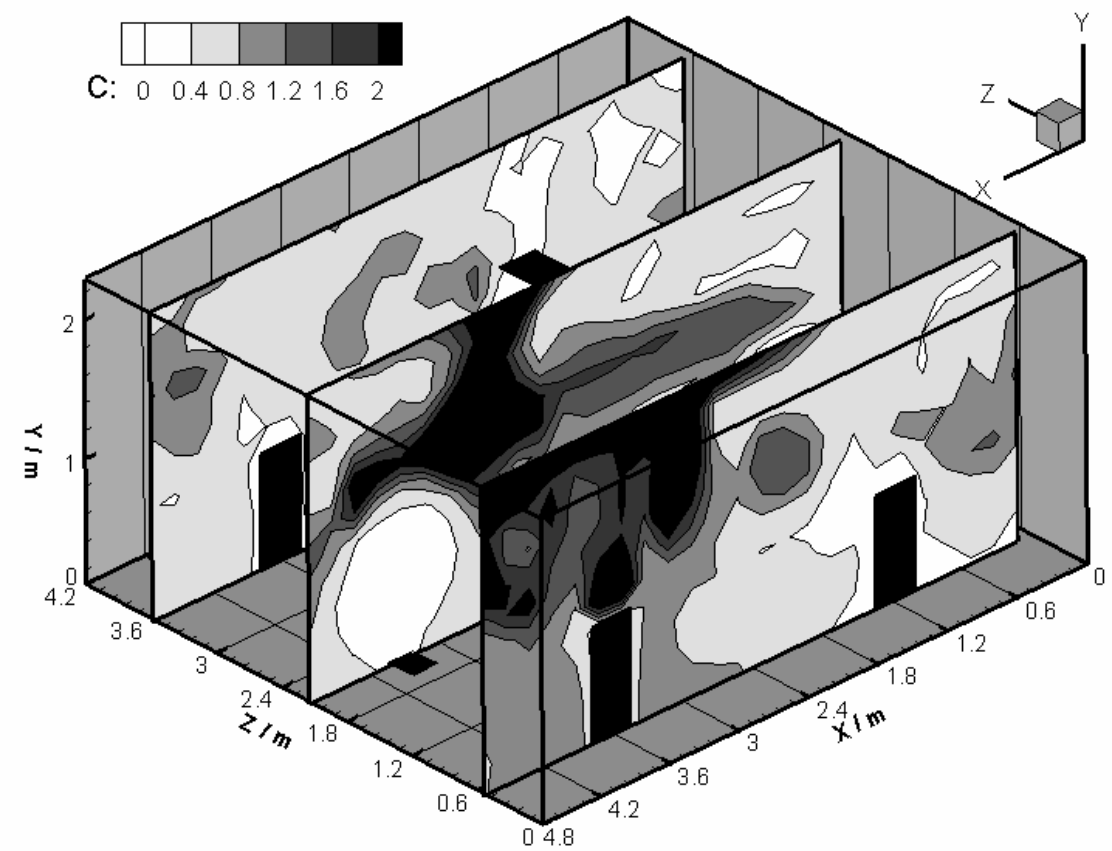

Fig. 10. Particle concentration distribution with the source near an occupant (S2) in the room with UFAD system. 